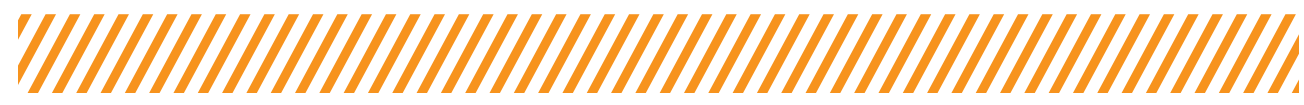

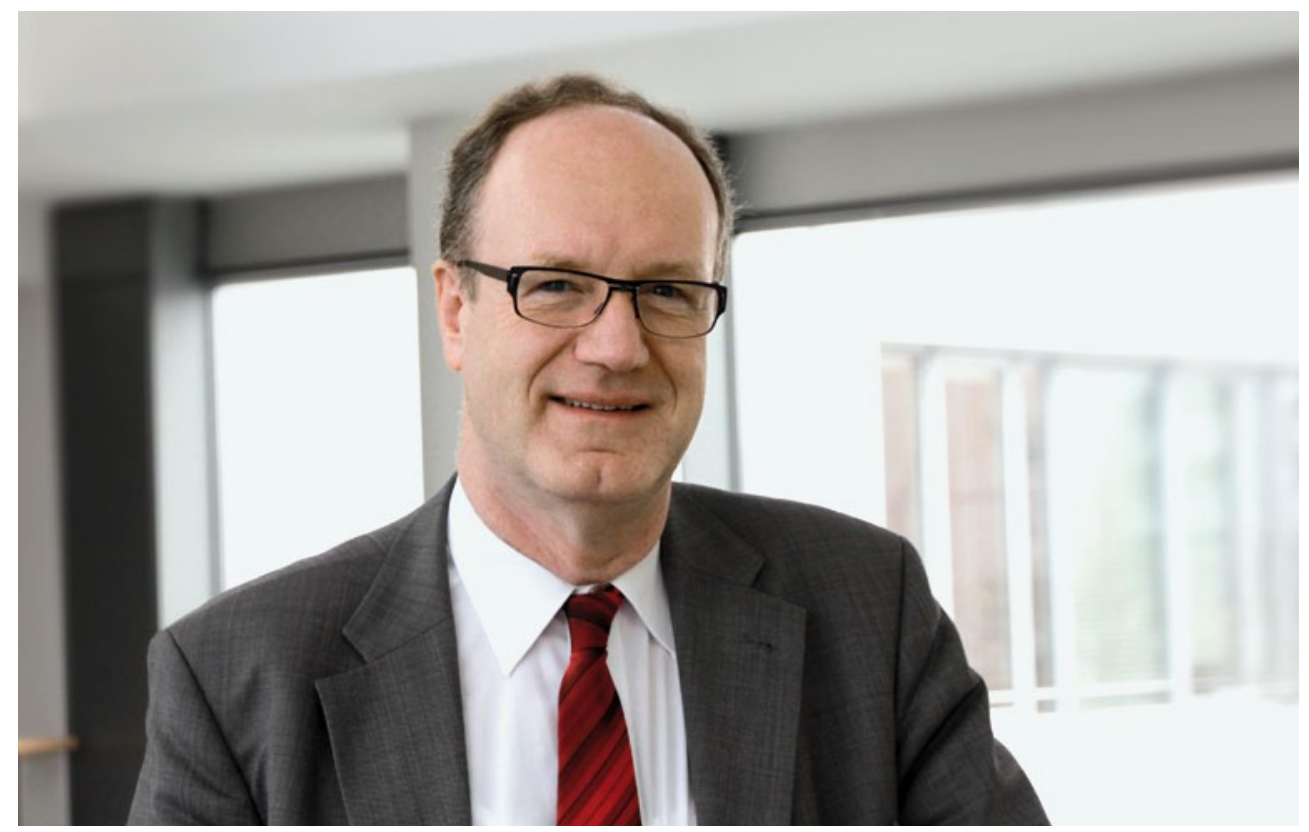

HANS-GEORG FRISCHKORN

Mitglied des Wissenschaflichen Beirats der ATZelektronik und

Beirat des 1. Internationalen ATZ-

Fachkongress Fahrassistenzsysteme

\section{NEUE TESTMETHODEN FÜR FAHRERASSISTENZSYSTEME}

Die zunehmenden Ausstattungsraten von Fahrerassistenzsystemen erzeugen eine enorme funktionale Vielfalt und Komplexität, die verstärkt auch sicherheitsrelevante Systeme wie den Notbremsassistent, den Kreuzungs- oder auch Überholassistent beinhalten. Mit der Einführung von fahrzeugübergreifend kommunizierenden Assistenzfunktionen und hochautomatisierten Fahrfunktionen wächst die Komplexität nochmals exorbitant. Die Absicherung solcher hochvernetzter Fahrfunktionen stellt neue und extrem hohe Anforderungen an den Testprozess - dieser Herausforderung muss sich die Automobilindustrie stellen.

Die Absicherung von Automatisierungsfunktionen ist in hohem Maße abhängig von stochastischen Einflüssen in einem mehrdimensional und komplexen Parameterraum. Diese Komplexität macht vollständige Systemtests im klassischen Sinne absolut unmöglich.

Wie geht man damit um? Das Testen von Assistenzfunktionen wird derzeit in der Regel mit den klassischen Ansätzen MiL/SiL, HiL und Fahrzeugerprobung durchgeführt. Das Augenmerk ist hier insbesondere auf eine direkte Kausalität zwischen den ausgewählten technischen Stimuli und dem resultierenden Verhalten im Testaufbau gerichtet. Im Idealfall leiten sich die entsprechenden Ursache-Wirkung-Beziehungen als Testfälle aus den formulierten Anforderungen oder der Systemspezifikation ab. Im Hinblick auf die Verifikation von kooperativen und hochautomatisierten Funktionen stoßen diese
Absicherungskonzepte aber an Grenzen. Stattdessen bedarf es einer Absicherungsmethodik, die dem nicht-deterministischen Charakter des Gesamtsystems - Umwelt, Fahrzeugsensorik und Autonomiefunktion/Fahrer - Rechnung trägt.

Ausgehend von diesen Überlegungen leiten sich drei grundlegenden Merkmale eines neuen Test- und Verifikationsprozesses ab: holistische Systembetrachtung, systematisches Ausloten der Systemgrenzen und Reproduzierbarkeit.

Ein möglicher Absicherungsansatz aus der Forschung ist die sogenannte formale Verifikation. Die diesbezüglich beschriebenen methodischen Konzepte erscheinen derzeit für den praktischen Einsatz noch nicht hinreichend weit entwickelt. Grundsätzlich liegt darin aber ein hohes Potenzial. Denn hier geht es um die Anwendung von formalen Methoden entlang der gesamten Wirkkette, von der Anforderungsspezifikation bis zum Nachweis der Funktionalität im Fahrzeug.

In jedem Fall - mit oder ohne Anwendung formaler Methoden - ist die Vollständigkeit und Exaktheit der Spezifikationen sowie ein konsequent gelebtes Änderungsmanagement auf Anforderungsebene eine entscheidende Voraussetzung, um die aufgezeigten neuen Herausforderungen erfolgreich anzugehen. Der Quantensprung von den klassischen regelbasierten Systemen zu komplexen Umfeldmodellen und darauf basierenden Bewertungs- und Entscheidungsmodellen ist anders nicht $\mathrm{zu}$ bewältigen. 